\title{
Partitioning complete graphs by heterochromatic trees *
}

\author{
Zemin $\mathrm{Jin}^{1}$ and Xueliang $\mathrm{Li}^{2}$ \\ ${ }^{1}$ Department of Mathematics, Zhejiang Normal University \\ Jinhua 321004, P.R. China \\ ${ }^{2}$ Center for Combinatorics and LPMC, Nankai University \\ Tianjin 300071, P.R. China
}

November 20, 2018

\begin{abstract}
A heterochromatic tree is an edge-colored tree in which any two edges have different colors. The heterochromatic tree partition number of an $r$-edge-colored graph $G$, denoted by $t_{r}(G)$, is the minimum positive integer $p$ such that whenever the edges of the graph $G$ are colored with $r$ colors, the vertices of $G$ can be covered by at most $p$ vertex-disjoint heterochromatic trees. In this paper we determine the heterochromatic tree partition number of an $r$-edge-colored complete graph.
\end{abstract}

Keywords: edge-colored graph, heterochromatic tree, partition.

AMS subject classification (2000): 05C05, 05C15, 05C70, 05C75.

\section{Introduction}

A monochromatic (heterochromatic) tree is an edge-colored tree in which any two edges have the same (different) color(s). The (monochromatic) tree partition number of an $r$-edge-colored graph $G$ is defined to be the minimum positive integer $p$ such that whenever the edges of $G$ are colored with $r$ colors, the vertices of $G$ can be covered by at most $p$ vertex-disjoint monochromatic trees. The (monochromatic) cycle partition number and the (monochromatic) path partition number are defined similarly.

Erdős, Gyárfás and Pyber [2] proved that the (monochromatic) cycle partition number of an $r$-edge-colored complete graph $K_{n}$ is at most $c r^{2} \ln r$

\footnotetext{
*Supported by NSFC, PCSIRT and the "973" program.
} 
for some constant $c$. This implies a conjecture from [5] in a stronger form. Recently, the bound was improved by Gyárfás et al. [6]. Almost solving one of the conjectures in [2], Haxell and Kohayakawa [8] proved that the (monochromatic) tree partition number of an $r$-edge-colored complete graph $K_{n}$ is at most $r$ provided that $n$ is large enough with respect to $r$. Haxell [7] proved that the (monochromatic) cycle partition number of an $r$ edge-colored complete bipartite graph $K_{n, n}$ is also independent of $n$, which answered a question in [2].

From above, one can see that the (monochromatic) tree, path, and cycle partition number of $r$-edge-colored graphs $K_{n}$ and $K_{n, n}$ are independent of $n$. The same seems to be not true for other graphs. Also, no (monochromatic) partition number of an $r$-edge-colored graph $K_{n}$ or $K_{n, n}$ is determined exactly. The only exception is due to Kaneko, Kano and Suzuki [9], who gave an explicit expression for the (monochromatic) tree partition number of a 2-edge-colored complete multipartite graph. In particular, let $n_{1}, n_{2}, \cdots, n_{k}(k \geq 2)$ be integers such that $1 \leq n_{1} \leq n_{2} \leq \cdots \leq n_{k}$ and let $n=n_{1}+n_{2}+\cdots+n_{k-1}, m=n_{k}$. The authors [9] proved that

$$
t_{2}^{\prime}\left(K_{n_{1}, n_{2}, \cdots, n_{k}}\right)=\left\lfloor\frac{m-2}{2^{n}}\right\rfloor+2,
$$

where $t_{r}^{\prime}\left(K_{n_{1}, n_{2}, \cdots, n_{k}}\right)$ denotes the (monochromatic) tree partition number of the $r$-edge-colored graph $K_{n_{1}, n_{2}, \cdots, n_{k}}$. Other related partition problems can be found in [3, 10, 11].

Analogous to the monochromatic tree partition case, the authors [1] introduced the definition of heterochromatic tree partition number of an $r$ edge-colored graph $G$. The heterochromatic tree partition number of an $r$-edge-colored graph $G$, denoted by $t_{r}(G)$, is defined to be the minimum positive integer $p$ such that whenever the edges of the graph $G$ are colored with $r$ colors, the vertices of $G$ can be covered by at most $p$ vertex-disjoint heterochromatic trees. In [1], the authors determined the heterochromatic tree partition number of an $r$-edge-colored complete bipartite graph $K_{m, n}$. In this paper we consider an $r$-edge-colored complete graph $K_{n}$ and give the exact expression for its heterochromatic tree partition number.

Before proceeding, we introduce some definitions and notations. Throughout this paper, we use $r$ to denote the number of the colors, and an $r$-edgecoloring of a graph $G$ means that each color appears at least once in $G$. Let $\phi$ be an $r$-edge-coloring of a graph $G$. For an edge $e \in E(G)$, denote by $\phi(e)$ the color of $e$. Denote by $t_{r}(G, \phi)$ the minimum positive integer $p$ such that under the $r$-edge-coloring $\phi$, the vertices of $G$ can be covered by at most $p$ vertex-disjoint heterochromatic trees. Clearly, $t_{r}(G)=\max _{\phi} t_{r}(G, \phi)$, where $\phi$ runs over all $r$-edge-colorings of the graph $G$. Let $\phi$ be an $r$-edge-coloring 
of the graph $G$ and $F$ be a spanning forest of $G$, each component of which is a heterochromatic tree. If $F$ contains exactly $t_{r}(G, \phi)$ components, then $F$ is called an optimal heterochromatic tree partition of the graph $G$ with edgecoloring $\phi$. Note that a tree consisting of a single vertex is also regarded as a heterochromatic tree.

For any integer $r \geq 2$, there is a unique positive integer $t$, such that $\left(\begin{array}{l}t \\ 2\end{array}\right)+2 \leq r \leq\left(\begin{array}{c}t+1 \\ 2\end{array}\right)+1$. Clearly, the integer $t$ is determined completely by $r$, and here we denote it by $f(r)=t$. This integer $f(r)=t$ will play an important role in expressing the number $t_{r}\left(K_{n}\right)$. If the color number $r=1$, clearly a maximum matching (plus a single vertex when $n$ is odd) in $K_{n}$ is an optimal heterochromatic tree partition, and then $t_{r}\left(K_{n}\right)=\left\lceil\frac{n}{2}\right\rceil$. So, in the rest of this paper we only consider the case $2 \leq r \leq\left(\begin{array}{l}n \\ 2\end{array}\right)$. The following is the main result of this paper.

Theorem 1.1 Let $n \geq 3,2 \leq r \leq\left(\begin{array}{l}n \\ 2\end{array}\right)$ and $f(r)=t$. Then $t_{r}\left(K_{n}\right)=\left\lceil\frac{n-t}{2}\right\rceil$.

As we know, the monochromatic tree partition number of an edge-colored complete graph $K_{n}$ is bounded by a function independent of $n$, and from the result mentioned above, the heterochromatic tree partition number does not have this property any more. The rest of the paper is organized as follows. In Section 2, we present a canonical $r$-edge-coloring $\phi^{*}$ of the complete graph $K_{n}$, and show that, under the canonical $r$-edge-coloring $\phi^{*}$, the optimal heterochromatic tree partition in the graph $K_{n}$ contains exactly $\left\lceil\frac{n-t}{2}\right\rceil$ components. The proof of our main result is complete in the last section.

\section{A canonical $r$-edge-coloring $\phi_{r}^{*}$}

In this section we present a canonical $r$-edge-coloring of the graph $K_{n}$. Let $f(r)=t$, i.e., $\left(\begin{array}{l}t \\ 2\end{array}\right)+2 \leq r \leq\left(\begin{array}{c}t+1 \\ 2\end{array}\right)+1$. Let $S \subseteq V\left(K_{n}\right)$ and $|S|=t$. Take a vertex $u \in V\left(K_{n}\right)-S$. We define the canonical $r$-edge-coloring $\phi_{r}^{*}$ by

1. giving distinct colors to the edges of $K_{n}[S]$;

2. for each color not used, assign it to an edge $u v$ (if it is not colored) between $u$ and $S$;

3. finally, color all the remaining edges by the color not used if it exists, or else by the same color.

We have the following proposition.

Proposition $2.1 t_{r}\left(K_{n}, \phi_{r}^{*}\right)=\left\lceil\frac{n-t}{2}\right\rceil$. 
Proof: First, we present an heterochromatic tree partition with exact $\left\lceil\frac{n-t}{2}\right\rceil$ components, which implies that $t_{r}\left(K_{n}, \phi_{r}^{*}\right) \leq\left\lceil\frac{n-t}{2}\right\rceil$. Let $X=S \cup\{u\} \cup\{v\}$, where $v \in V\left(K_{n}\right)-S-u$. It is easy to see that $K_{n}[X]$ contains a heterochromatic spanning tree $T$, and the vertices not in $X$ induce a monochromatic complete subgraph which can be covered by $\left\lceil\frac{n-t-2}{2}\right\rceil$ disjoint heterochromatic trees. So, the union of $T$ and those $\left\lceil\frac{n-t-2}{2}\right\rceil^{2}$ disjoint heterochromatic trees consist of a heterochromatic tree partition of $K_{n}$. This implies that $t_{r}\left(K_{n}, \phi_{r}^{*}\right) \leq\left\lceil\frac{n-t}{2}\right\rceil$.

Next, we prove that $t_{r}\left(K_{n}, \phi_{r}^{*}\right) \geq\left\lceil\frac{n-t}{2}\right\rceil$. Suppose on the contrary that $t_{r}\left(K_{n}, \phi_{r}^{*}\right)<\left\lceil\frac{n-t}{2}\right\rceil$ for some $n$ and $r$.

Let $F$ be an optimal heterochromatic tree partition of $K_{n}$ with $r$-edgecoloring $\phi_{r}^{*}$. Denote by $T_{1}, T_{2}, \cdots, T_{k}$ the components of $F$ which contains vertices of $S$. We choose $F$ such that the number of trees covering $S$ is as small as possible. Note that each component of $F$ not containing any vertex of $S$ is an edge or a single vertex, and at most one of the components of $F$ is a single vertex. Since $F$ is an optimal heterochromatic tree partition, from the definition of $\phi_{r}^{*}$, we have the following facts.

Fact 1. $u \in T_{i}$ for some $1 \leq i \leq k$.

Fact 2. $\left|T_{j} \cap\left(V\left(K_{n}\right)-S-u\right)\right|=1$ for each $T_{j}$.

If $k=1$, it is easy to see that $F$ just contains $\left\lceil\frac{n-t}{2}\right\rceil$ trees. So, assume that $k \geq 2$. Let $S \cap T_{i}=S_{i}$ and $v_{i}=T_{i} \cap\left(V\left(K_{n}\right)-S-u\right)$. From the definition of $\phi_{r}^{*}$, we have that there exists a heterochromatic tree, denoted by $T$, covering all the vertices $T_{1} \cup\left(T_{2}-v_{2}\right)$. So $F^{\prime}=\left(F-T_{1}-T_{2}\right) \cup\{T\} \cup\left\{v_{2}\right\}$ is an optimal heterochromatic tree partition such that the number of trees covering $S$ is $k-1$, a contradiction, which completes the proof.

\section{Proof of Theorem 1.1}

Given a complete graph $K_{n}$, the heterochromatic tree partition number is closely related to the color number. Before proving our main result, we have the following lemma which presents the relationship between $t_{r+1}\left(K_{n}\right)$ and $t_{r}\left(K_{n}\right)$.

Lemma $3.1 t_{r+1}\left(K_{n}\right) \leq t_{r}\left(K_{n}\right)$.

Proof: Given any $(r+1)$-edge-coloring $\varphi$ of $K_{n}$. Denote by $E_{i}$ the set of edges colored by the color $i$. Recoloring the edges of $E_{r+1}$ by the color $r$, we obtain a $r$-edge-coloring $\psi$ of $K_{n}$. Clearly, $t_{r+1}\left(K_{n}, \varphi\right) \leq t_{r}\left(K_{n}, \psi\right)$. So, $t_{r+1}\left(K_{n}\right) \leq t_{r}\left(K_{n}\right)$. 
The following lemma presents the relationship between the edge-connectivity and size of a graph. The proof is omitted here.

Lemma 3.2 Let $G$ be a simple graph of order $n$. If $G$ contains a cut-edge, then $|E(G)| \leq\left(\begin{array}{c}n-1 \\ 2\end{array}\right)+1$.

Proof of Theorem 1.1: We prove the theorem by induction on $r$ and $n$. First, we consider the case $r=2$. Let $\phi$ be a 2-edge-coloring of $K_{n}$. Note that for any 2-edge-coloring of $K_{n}, n \geq 3$, there is always a heterochromatic tree of order three. Then, we can easily find $1+\left\lceil\frac{n-3}{2}\right\rceil=\left\lceil\frac{n-1}{2}\right\rceil$ vertex-disjoint heterochromatic trees which cover all the vertices. So we have $t_{r}\left(K_{n}, \phi\right) \leq\left\lceil\frac{n-1}{2}\right\rceil$. Then, from Proposition 2.1 the result holds for $r=2$. Obviously, the result holds for $n=3,4$.

Assume that the result holds for the color number less than $r$ or the order of a complete graph less than $n$. Now we consider the $r$-edge-colored complete graph $K_{n}, r \geq 3$. Let $f(r)=t$. If $\left(\begin{array}{c}t \\ 2\end{array}\right)+3 \leq r \leq\left(\begin{array}{c}t+1 \\ 2\end{array}\right)+1$, then $f(r-1)=t$. By the induction hypothesis, $t_{r-1}\left(K_{n}\right)=\left\lceil\frac{n-t}{2}\right\rceil$. From Lemma [3.1, $t_{r}\left(K_{n}\right) \leq t_{r-1}\left(K_{n}\right)=\left\lceil\frac{n-t}{2}\right\rceil$. And, from Proposition 2.1. $t_{r}\left(K_{n}\right) \geq t_{r}\left(K_{n}, \phi_{r}^{*}\right)=\left\lceil\frac{n-t}{2}\right\rceil$. Then, we have $t_{r}\left(K_{n}\right)=\left\lceil\frac{n-t}{2}\right\rceil$, as desired.

So, we only need to consider the case $r=\left(\begin{array}{l}t \\ 2\end{array}\right)+2$. Let $\phi$ be an $r$ edge-coloring of $K_{n}$. Let $G$ be a heterochromatic subgraph of $K_{n}$, such that $\delta(G) \geq 1$ and, for each color $i$, there is a unique edge colored by the color $i$ in $G$. Denote by $G_{1}, G_{2}, \cdots, G_{k}$ the components of $G$, where the order of $G_{i}$ is $n_{i}, 1 \leq i \leq k$, and $n_{1} \geq n_{2} \geq \cdots \geq n_{k} \geq 2$. Choose $G$ such that $n_{1}$ is as large as possible. Since the color number $r \geq 3$, we have $n_{1} \geq 3$.

Suppose that $k=1$. By $r=\left(\begin{array}{l}t \\ 2\end{array}\right)+2$, we have $n_{1} \geq t+1$. If $n_{1} \geq t+2$, then $t_{r}\left(K_{n}, \phi\right) \leq 1+\left\lceil\frac{n-n_{1}}{2}\right\rceil \leq\left\lceil\frac{n-t}{2}\right\rceil$. So, assume $n_{1}=t+1$. By Lemma 3.2. $G$ does not contain any cut-edge. Let $g \in\left[V\left(G_{1}\right), \overline{V\left(G_{1}\right)}\right]$, i.e., one endvertex of $g$ belongs to $V\left(G_{1}\right)$ and the other one belongs to $\left.\overline{V\left(G_{1}\right)}\right]$. From the choice of $G$, there is an edge $h \in E\left(G_{1}\right)$ with $\phi(h)=\phi(g)$. Since $G$ does not contain any cut-edge, by deleting the edge $h$ and adding the edge $g$, we can find a heterochromatic graph with $r$ edges, the largest component of which has an order at least $n_{1}+1$. A contradiction to the choice of the graph $G$.

So, assume $k \geq 2$. If $n_{1} \geq t+2$, then $t_{r}\left(K_{n}, \phi\right) \leq 1+\left\lceil\frac{n-n_{1}}{2}\right\rceil \leq\left\lceil\frac{n-t}{2}\right\rceil$, as desired. Thus, assume $n_{1} \leq t+1$. We have the following claim.

Claim: $G_{1}$ contains a cut-edge, and then $\left|E\left(G_{1}\right)\right| \leq\left(\begin{array}{c}n_{1}-1 \\ 2\end{array}\right)+1$.

Otherwise, suppose that $G_{1}$ does not contain any cut-edge. Let $g \in$ $\left[V\left(G_{1}\right), \overline{V\left(G_{1}\right)}\right]$. From the choice of $G$, there is an edge $h \in E\left(G_{1}\right)$ with 
$\phi(h)=\phi(g)$. Since $G$ does not contain any cut-edge, by deleting the edge $h$ and adding the edge $g$, we can find a heterochromatic graph with $r$ edges, the largest component of which has an order at least $n_{1}+1$, a contradiction to the choice of the graph $G$. From Lemma 3.2, $\left|E\left(G_{1}\right)\right| \leq\left(\begin{array}{c}n_{1}-1 \\ 2\end{array}\right)+1$ follows clearly. This completes the proof of the claim.

Now we consider the graph $K_{n}-V\left(G_{1}\right)$, a complete graph of order $n-n_{1}$. When restricting the $r$-edge-coloring $\phi$ on the graph $K_{n}-V\left(G_{1}\right)$, we have that $K_{n}-V\left(G_{1}\right)$ is edge-colored by $r_{0}$ colors, where $r_{0} \geq r-\left(\left(\begin{array}{c}n_{1}-1 \\ 2\end{array}\right)+1\right)$. If $r_{0} \geq 2$, let $f\left(r_{0}\right)=t_{0}$. It follows that either $r_{0}=1$, or $t_{0} \geq t-n_{1}+1$. We distinguish the following cases.

Case 1. $r_{0}=1$.

Then $K_{n}-V\left(G_{1}\right)$ is monochromatic, and then it follows that $k=2$ and $n_{2}=2$. Let $G_{2}=u v$. From the choice of $G$, we have $\left|E\left(G_{1}\right)\right|=r-1=$ $\left(\begin{array}{l}t \\ 2\end{array}\right)+1$. By $n_{1} \leq t+1$, we have $n_{1}=t+1$. From Claim 1 , let $e$ be a cut-edge in $G_{1}$. Since $\left|E\left(G_{1}\right)\right|=\left(\begin{array}{l}t \\ 2\end{array}\right)+1$ and $n_{1}=t+1$, we have $G_{1}-e \cong K_{t} \cup K_{1}$. Let $w \in V\left(G_{1}\right)$. From the choice of $G$, we have $\phi(u w) \neq \phi(u v)$, and there is a cut-edge in $G_{1}$ colored by the same color $\phi(u w)$.

If $n_{1} \geq 4$, from $G_{1}-e \cong K_{t} \cup K_{1}$, we have that $e$ is the unique cut-edge in $G_{1}$. By $G_{1}-e \cong K_{t} \cup K_{1}$, we can take a vertex $w$ which is not single in $G_{1}-e$. Then $\phi(u w)=\phi(e)$. By deleting the edge $e$ and adding the edge $u w$, we can find a heterochromatic graph with $r$ edges, the largest component of which has an order at least $n_{1}+1$, a contradiction to the choice of $G$.

So, assume $n_{1}=3$. Then $r=3$ and $G_{1} \cong P_{3}$. Let $G_{1}=x y z$. Then either $\phi(y u)=\phi(x y)$ or $\phi(y u)=\phi(y z)$. Without loss of generality, assume $\phi(y u)=\phi(x y)$. Then $\phi(y u) \neq \phi(y z)$. Again, the graph $z y u v$ is heterochromatic and of size $r$, a contradiction to the choice of $G$.

Case 2. $t_{0} \geq t-n_{1}+1$.

Since $r_{0} \geq 2$, we have $t_{0} \geq 1$. If $t_{0} \geq t-n_{1}+2$, then by the induction hypothesis, the graph $K_{n}-V\left(G_{1}\right)$ can be covered by at most $\left\lceil\frac{n-n_{1}-t_{0}}{2}\right\rceil$ vertexdisjoint heterochromatic trees. Thus, $t_{r}\left(K_{n}, \phi\right) \leq 1+\left\lceil\frac{n-n_{1}-t_{0}}{2}\right\rceil \leq\left\lceil\frac{n-t}{2}\right\rceil$, as desired.

Suppose $t_{0}=t-n_{1}+1$. Then we have $r=\left(\begin{array}{l}t \\ 2\end{array}\right)+2 \leq\left|E\left(G_{1}\right)\right|+r_{0} \leq$ $\left(\begin{array}{c}n_{1}-1 \\ 2\end{array}\right)+1+\left(\begin{array}{c}t_{0}+1 \\ 2\end{array}\right)+1=\left(\begin{array}{c}n_{1}-1 \\ 2\end{array}\right)+1+\left(\begin{array}{c}t-n_{1}+1+1 \\ 2\end{array}\right)+1$. This implies that $\left(\begin{array}{l}t \\ 2\end{array}\right) \leq\left(\begin{array}{c}n_{1}-1 \\ 2\end{array}\right)+\left(\begin{array}{c}t-\left(n_{1}-1\right)+1 \\ 2\end{array}\right)$, i.e., $\left(n_{1}-1\right)\left(t-\left(n_{1}-1\right)\right) \leq t-\left(n_{1}-1\right)$. By $n_{1} \geq 3$ and $n_{1} \leq t+1$, we have $n_{1}=t+1$, and then $t_{0}=0$, a contradiction to the fact $t_{0} \geq 1$. The proof is now complete. 


\section{References}

[1] H. Chen, Z.M. Jin, X.L. Li and J.H. Tu, Heterochromatic tree partition number of complete bipartite graphs, submitted, 2006.

[2] P. Erdős, A. Gyárfás and L. Pyber, Vertex coverings by monochromatic cycles and trees, J. Combin. Theory Ser.B 51(1991), 90-95.

[3] P. Erdős and A. Gyárfás, Split and balanced colorings of complete graphs, Discrete Math. 200(1999), 79-86.

[4] A. Gyárfás, Vertex coverings by monochromatic paths and cycles, $J$. Graph Theory 7(1983), 131-135.

[5] A. Gyárfás, Covering complete graphs by monochromatic paths, in "Irregularities of Partitions," Algorithms and Combinatorics, Vol.8, Springer-Verlag, 1989, pp. 89-91.

[6] A. Gyárfás, M. Ruszinkó, G.N. Sárközy and E. Szemerédi, An improved bound for the monochromatic cycle partition number, J. Combin. Theory Ser.B 96(2006), 855-873.

[7] P.E. Haxell, Partitioning complete bipartite graphs by monochromatic cycles, J. Combin. Theory Ser.B 69(1997), 210-218.

[8] P.E. Haxell and Y. Kohayakawa, Partitioning by monochromatic trees, J. Combin. Theory Ser.B 68(1996), 218-222.

[9] A. Kaneko, M. Kano and K. Suzuki, Partitioning complete multipartite graphs by monochromatic trees, J. Graph Theory 48(2005), 133-141.

[10] T. Luczak, V. Rödl and E. Szemerédi, Partitioning 2-edge-colored complete graphs into 2 monochromatic cycles, Combinatorics, Probability and Computing 7(1998), 423-436.

[11] R. Rado, Monochromatic paths in graphs, Annals of Discrete Math. 3(1978), 191-194. 